\title{
Self-adaptiveness in particle swarm optimisation to enhance available transfer capability using thyristor-controlled series compensation (TCSC)
}

\author{
NARESH KUMAR YADAV* and ANJU BALA \\ Department of Electrical Engineering, DCR University of Science and Technology, Murthal (Sonepat), India \\ e-mail: nareshyadhavdr@gmail.com
}

MS received 1 August 2017; revised 12 October 2017; accepted 16 October 2017; published online 1 August 2018

\begin{abstract}
Available transfer capability (ATC) is one of the challenging criteria under the functioning of the deregulated power system. The high demand for improving ATC is generally met using flexible alternating current transmission system (FACTS) devices in the power system. However, it suffers from serious crisis during determination of the optimal location and compensation stage of FACTS. The present study uses thyristor-controlled series compensation (TCSC) devices in order to compensate for the limitation of FACTS. Further, a novel self-adapted particle swarm optimisation (SAPSO) algorithm is proposed in this study for enhancing ATC. Experiments are carried on three benchmark bus systems such as IEEE 24, IEEE 30 and IEEE 57. Performance and statistical analyses are carried out by comparing the proposed SAPSO with the conventional PSO. Eventually, the study proves the effectiveness of the proposed method in case of ATC enhancement.
\end{abstract}

Keywords. Power system; ATC; TCSC; SAPSO.

\section{Introduction}

The problems associated with the power system are solved using knowledge-based decision-making approach by majority of scientists till date. Among such promising methods, the artificial intelligent method plays a vital role in solving problems in the power system [1,2]. There are diverse techniques such as bio-inspired optimisation algorithms, swarm intelligence, and Chaos theory that are classified as Artificial Intelligence (AI) algorithms [3-5]. Available Transfer Capability (ATC) is considered as the main factor in power system $[1,6]$. Since there is a limitation in power system due to the reorganisation of the power system and rise of startling in demand of power, the system should be operated under the standard limits [7-9]. Furthermore, security and reliability of the power system is highly increased with ATC enhancement [10].

Currently, there is a limitation for the enhancement of ATC [11, 12], because broadening of the transmission network destroys the network [13]. Furthermore, these problems are solved by the flexible alternating current transmission system (FACTS) devices with improved strength as these devices handle the rearrangement of flow of lines and control of voltage [2,14-16]. However, it is not cost-efficient $[6,15]$. Since the FACTS devices are highly rewarding, they are mainly used for the purpose of ATC

*For correspondence enhancement [17-20]. Therefore, solving the problem associated with the improvement of ATC appears to be an individual optimisation problem or individual decisionmaking problem or both. Optimisation problems are generally categorised as multi-objective optimisation and single-objective optimisation [4]. Amid the diverse types, the proposed SAPSO plays a vital role in solving the optimisation problems under the power system [21, 22], which is implemented in this study.

\section{Literature review}

\subsection{Related works}

Several recent methodologies related to the optimisation of the FACTS devices have been developed. Initially, the ATC was improved by Rashidinejad et al [23] effectively using the real genetic algorithm (GA). Further, Nireekshana et al [10] have proposed the novel technique using a few FACTS devices such as thyristor-controlled series compensation (TCSC) and Static Var Compensator in order to enhance the ATC. In the aforementioned work, real coded GA was used so that the compensating reactance and most advantageous location related with FACTS devices was selected. Experiments were performed on both IEEE 14-bus system and IEEE 24-bus system under emergency as well as normal conditions. Accordingly, Farahmand et al [20] 
developed hybrid PSO to improve the ATC. This algorithm has been adapted greatly to solve multi-objective problems, and the experiment was carried out in IEEE 30-bus system and IEEE 57-bus system. Further, the Bacterial Swarm Optimisation (BSO)-based ATC improvement method was implemented by Ali and Abd-Elazim [6]. Similarly, the total transfer capability (TTC) was enhanced using a probabilistic-based method that extracted the features of the probabilistic modelling in a study by Khaburi and Haghifam [24]. The improvement of TTC directly enhances the ATC. Although the methods mentioned earlier have the advantage of enhancing the ATC, they suffer from the diverse drawbacks that affect the operation of the power system in case of improving the ATC [25-27]. Hence, it is difficult to attain the objective due to progressive demand in power system. Further, the convergence speed of the operation is highly affected by the cost [23] and the multiobjective problems arising in the functioning of the power system are complex [20]. Arijit De et al [28] formulated a model by exploiting the PSO-CP (Composite Particle) method. The proposed PSO-CP assists in escaping from the local optima and consistently achieving near-optimal point. Alexandridis et al [29] presented a cooperative PSO framework which permits for exploiting variable-width basis functions. This method was utilised to avoid the excessive number of design variables. De et al [30] presented an effective search heuristics named PSO-CP method, and a mathematical model is developed to address a maritime inventory routing problem. Hamed Soleimani and Govindan Kannan [31] presented a comprehensive model and a novel PSO-GA hybrid method to solve several kinds of problems in the field of closed-loop supply chain (CLSC). De et al [32] worked on ship routing and scheduling problem, and this is formulated as a Mixed Integer Non-Linear Programming Model (MINLP) solved by PSO-CP.

\subsection{Research gaps}

Several researchers have worked extensively on optimisation of the FACTS devices. In ref. [23], a GA method has been developed to avoid being trapped into local optimal solutions. However, a GA method does not scale well with complexity. In refs. [20, 29, 31], a hybrid PSO has been developed to solve systems of non-linearity, and it can be used to overcome the difficulty of inaccuracy occuring in PSO. Nonetheless, it does not support multi-level linear programming problem. In contrast, a GA [97] has some advantages such as security, and it ensures large key space, but it is statistically more complex. The BSO used in ref. [6] shows that it can easily perform local search. Conversely, the convergence rate of this method is slow. Moreover, the probabilistic-based method used in Ref. [24] has shown its advantages such as ease of use and convenience, but it is time consuming. The PSO-CP approach was used in [28, 30, 32], which has maintained the entire population diversity by modeling all particles. However, it suffers from solving the numerical optimisation problems.

According to the review, earlier studies have the advantage of improving the ATC. However, there are several disadvantages affecting the operation of the power system in case of enhancing the ATC. Therefore, it is necessary to enhance the ATC using the advanced metaheuristic optimisation algorithm.

Contribution: The main contribution of this paper is to propose an SAPSO method to solve the optimisation problems under the power system. Also, the proposed method is exploited to optimise the maximum ATC in power transmission system. Moreover, TCSC devices are utilised in the present study instead of FACTS devices.

\section{Enhancement model of ATC}

\subsection{ATC basics}

As the requirement of electricity is increased, the producers and the consumers of the power transmission system are moving in a deregulated manner. So, a single transmission system of power is used for conveying power between the generator part and consumer part. In electricity market, the number of generators can be vary that leads to the occurrence of overcapacity and jamming. Since congestion and overloading are increased, there is destruction in the flow of transmission line and the stability limit and voltage limits are increased. These issues highly affect the ATC of the power system, as ATC greatly helps to provide protection and consistency to the system. Open Access Same Time Information System (OASIS) is kept to update the ATC continuously, therefore estimation of ATC becomes tedious part. By general definition, "ATC is a measure of the transfer capability remaining in the physical transmission network for further commercial activity over and above already committed uses." The common formulation of ATC is represented in Eq. (1), where $V^{E T C}$ represents the existing transmission commitments (ETC), $V^{T T C}$ represents the TTC, $V^{C B M}$ represents the capacity benefit margin (CBM), and $V^{T R M}$ represents the transfer reliability margin (TRM). The definition of ATC with the clear description of TTC, TRM, ETC and CBM is shown in figure 1.

$$
V^{A T C}=V^{T T C}-V^{T R M}-\left(V^{E T C}+V^{C B M}\right)
$$

The description related to the other parameters of ATC such as TRM, CBM and TTC follows.

TRM: TRM refers to the measure of transmission ability of the power transfer, which is required to establish the security of the interrelated system. For the above 


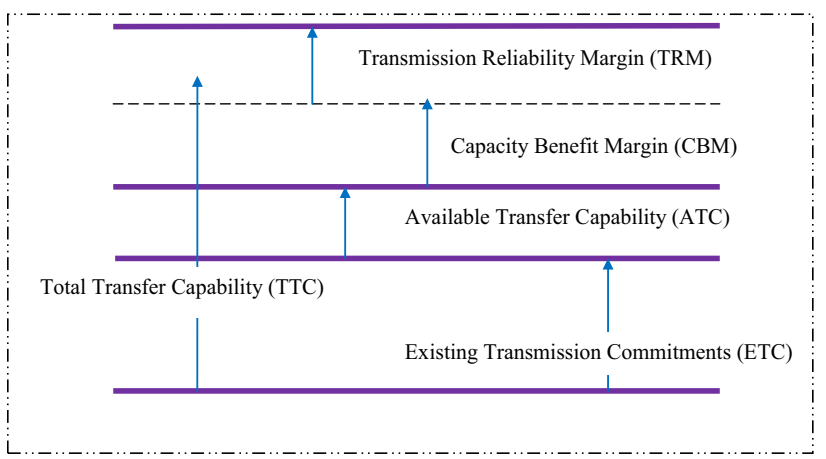

Figure 1. ATC definition.

measurement, the power system may be linked with few uncertainties that remain in the system.

CBM: CBM refers to the measure of transmission ability of power transfer by satisfying the needs of reliable generation. Here, the components available for easing the load are highly utilised to operate under the interconnected system.

TTC: TTC refers to the measure of the total power transferred through the system in such a way that the entire set of defined pre-contingency as well as post-contingency system conditions is satisfied.

\subsection{ATC determination}

There have been several experiments associated with the increment of ATC in the past few years, which have been reviewed by diverse researchers. For instance, the contour of the security boundary was considered by Li and Liu [33] for ATC determination. Further,Vaithilingam and Kumudini Devi [34] have estimated the ATC using the Support Vector Machine. Since the advent of techniques for the estimation of ATC has increased year by year, their fictional techniques are categorised into two types: (i) based on distribution factors and (ii) based on Continuous Power Flow (CPF) [35]. The aforementioned methods are used in case of DC or AC power flows. However, this experimentation is not effective as it does not need any complexity and breakdown to produce the accurate result for the overall operation for ATC determination [23]. Moreover, the CPF approaches are mainly adopted as they use the addition of linear variation at the class of buses to obtain the optimum value [3]. Therefore, the controlling parameters associated with the experimentation under CPF are moving in a linear manner. Thus, it requires the rectification process after the completion of each iteration. The present study completely depends on the power flow for the estimation of ATC.

\subsection{Objective function}

Let us consider a classic bus system connected with a few external devices. The ATC should be estimated for the power system. Subsequently, this study focuses on the estimation of ATC of the system accessible with TCSC. The requirement of external devices under such instances is less as the number of TCSC to be connected depends on the users. The objective problem model is represented in Eq. (2), where $A_{t}$ indicates the group of line indices and $B_{t}$ indicates the compensation level of $t^{\text {th }}$ TCSC in the bus system, $\left[A_{t}^{*}, B_{t}^{*}\right]$ represents the best connection in the group of line.

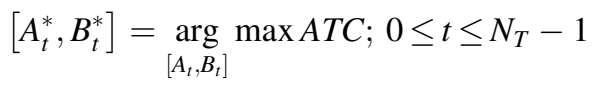

\section{ATC enhancement by SAPSO}

\subsection{Standard PSO}

PSO [21, 22] is the type of evolutionary algorithm introduced by Kennedy and Eberhart in 1995. The operation of PSO is based on the behaviour of flocking of birds to solve the optimisation problems. Thus, the PSO algorithm is named as the effective optimisation algorithm in real time. Starting with the execution of PSO algorithm, a random solution is initialised, and the optimum solution is searched through updating the generations. Each particle $P_{m}$ comprises the position vector, velocity vector and memory vector. The position vector is represented by $X_{m}$, the velocity vector is represented by $V_{m}$ and memory vector is represented by $X_{m}^{\text {best }}$. The solution associated with the problem of optimisation is provided by the position vector, whereas the best solution derived with the search of one particle is considered as the memory vector. The particle flies with particular velocity in the dimensional space, where the velocity is attuned according to the flying skill of the corresponding particle along with its colleagues. The particle may search for its better position though this operation. Subsequently, the particle is updated as in Eq. (3), whereas the updating of the velocity is represented in Eq. (4), where $r_{1}$ and $r_{2}$ represents the random variables that are equally distributed and in the range of $[0,1], c_{1}$ and $c_{2}$ represents the accelerating constants, $X_{g}^{\text {best }}$ represents the best solution searched by entire particles, and $w_{m}$ represents the inertia weight of the particle. The convergence speed of the algorithm completely depends on the inertia weight.

$$
\begin{gathered}
X_{m}(t+1)=X_{m}(t)+V_{m}(t+1) \\
V_{m}(t+1)=w_{m} V_{m}(t)+c_{1} r_{1}\left(X_{m}^{\text {best }}(t)-X_{m}(t)\right) \\
+c_{2} r_{2}\left(X_{g}^{\text {best }}(t)-X_{m}(t)\right)
\end{gathered}
$$

The pseudo-code of the standard PSO algorithm is illustrated as follows. 


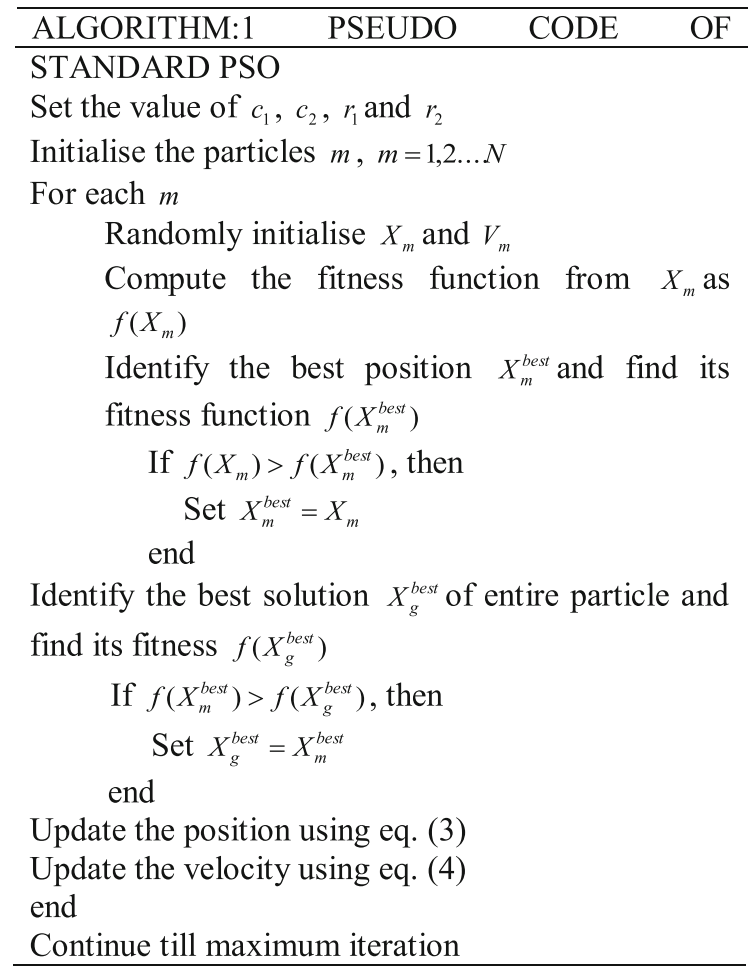

\subsection{Proposed SAPSO}

The SAPSO is an algorithm based on balancing the inertia weight, which is set as a self-improvement strategy. Here the inertia weight is updated based on the fitness function obtained at each position of the particles. Since the weight is updated, the adaptive solution is obtained, which satisfies the enhancement of ATC. The position of the particle in each iteration should be evaluated by adapting the inertia weight at each iteration. After completion of each iteration, the self-improvement ratio must be calculated, and the inertia weight should be increased with respect to the ratio. Computation of inertia weight based on the selfadaptive procedure is represented in Eq. (5), where $r_{m}(t+$ 1) represents the random variable distributed at $(t+1)^{t h}$ iteration and $\partial_{m}(t+1)$ represents the ratio associated with the self-improvement at $(t+1)^{t h}$ iteration, which is expressed in Eq. (6). The fitness function of $m^{\text {th }}$ particle at $t^{t h}$ and $(t-1)^{t h}$ iterations are denoted as $f\left(X_{m}(t)\right.$ and $f\left(X_{m}(t-1)\right.$. Accordingly, the final global fitness $f\left(X_{g}^{\text {best }}\right)$ is the ATC measurement.

$$
\begin{array}{r}
w_{m}(t+1)=w_{m}(t)+\left[r_{m}(t+1)\right. \\
*\left(w_{m}(t)-w_{m}(t-1) * \partial_{m}(t+1)\right] \\
\partial_{m}(t+1)=\frac{f\left(X_{m}(t)-f\left(X_{m}(t-1)\right.\right.}{f\left(X_{m}(t)\right.}
\end{array}
$$

The pseudo-code of the proposed SAPSO is illustrated as follows.

\section{ALGORITHM:1 PSEUDO CODE OF PROPOSED SAPSO}

1. Set the value of $c_{1}, c_{2}, r_{1}$ and $r_{2}$

2. Initialise the particles $m, m=1,2 \ldots N$

3. For each $m$

4. For first iteration $t=0$

5. $\quad$ Randomly initialise $w_{m}, X_{m}$ and $V_{m}$

6. $\quad$ Compute the fitness function from $X_{m}$ as $f\left(X_{m}\right)$

7. Identify the best position $X_{m}^{\text {best }}$ and find its fitness function $f\left(X_{m}^{\text {best }}\right)$

8. If $f\left(X_{m}\right)>f\left(X_{m}^{\text {best }}\right)$, then

9. $\quad$ Set $X_{m}^{\text {best }}=X_{m}$ end

10. Identify the best solution $X_{g}^{\text {best }}$ of entire particle and find its fitness $f\left(X_{g}^{\text {best }}\right)$

11. If $f\left(X_{m}^{\text {best }}\right)>f\left(X_{g}^{\text {best }}\right)$, then

12. Set $X_{g}^{\text {best }}=X_{m}^{\text {best }}$ end

end

13 Compute the self improvement ratio using eq.

(6)

14.

For second iteration $t=1$

15. Update the position using eq. (3)

16. Update the velocity using eq. (4)

18. Repeat the steps (6) to (13) end

19. Continue till maximum iteration

The flowchart of the SAPSO for the ATC enhancement is shown in figure 2.

The pseudo-code and the flowchart of SAPSO algorithm are elucidation as folows.

1. The accelerating constants $c_{1}, c_{2}$ and the random variables $r_{1}$ and $r_{2}$ are set in the initial stage.

2. The total number of particles is considered as $m$ and the number of particles should be initialised.

3. The position $X_{m}$ and velocity $V_{m}$ of the particle are initialised.

4. In the first iteration at $t=0$, the weight $w_{m}$ should be randomly initialised.

5. Subsequently, the best position $X_{m}^{\text {best }}$ is identified and along with it, the fitness function of $X_{m}^{\text {best }}$ is computed which is represented as $f\left(X_{m}^{\text {best }}\right)$.

6. If the fitness function of the current position is greater than the fitness function of the best position of the particle, then the best position is replaced by the current position.

7. Further, the best position of the entire particle $X_{g}^{\text {best }}$ is identified, and along with it, the fitness function of $X_{g}^{\text {best }}$ is computed that is represented as $f\left(X_{m}^{\text {best }}\right)$.

8. If the fitness function of the best position is greater than the fitness of the global best position, then the global best position is replaced by the best position. 


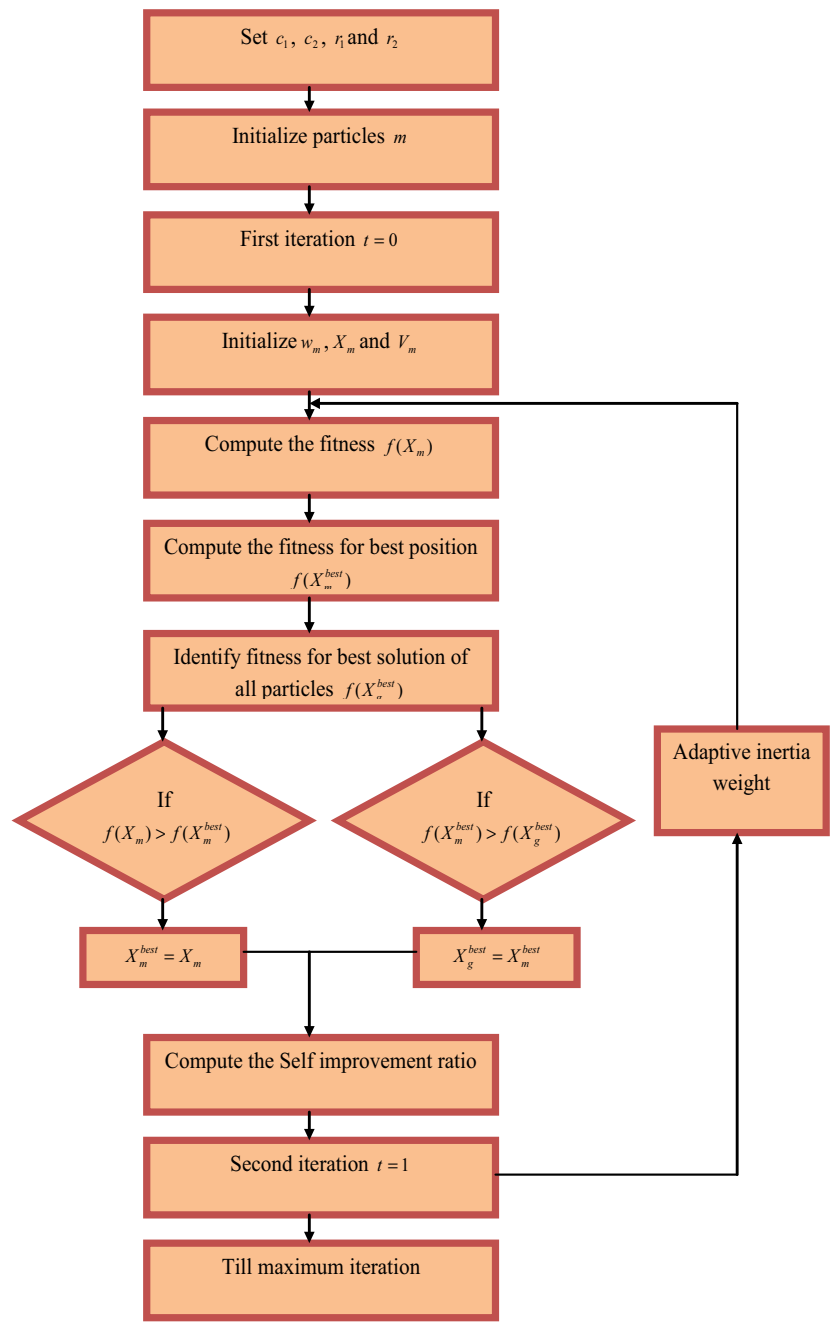

Figure 2. Flowchart of Self-Adaptive PS0 algorithm.
9. The self-improvement ratio $\partial_{m}(t+1)$ is calculated using Eq. (6) after the each iteration.

10. In the second iteration at $t=1$, the weight $w_{m}$ is improved using Eq. (5) along with the updating of the position and velocity of the particle using Eq. (3) and Eq. (4) and it repeats the steps from 4 to 8 .

11. The aforementioned procedure is repeated till the completion of an entire iteration.

\section{Results and discussions}

\subsection{Experimental procedure}

The experimentation regarding the ATC enhancement in power system was carried on IEEE 24, IEEE 30 and IEEE 57 benchmark test bus systems. The corresponding experimentation was performed using the proposed SAPSO. The result obtained from the proposed SAPSO was compared with that from the conventional PSO. Basically, the ATC enhancement was estimated under two TCSC connections as per the literature. However, this study indents to estimate under five TCSC connections. The statistical report of the three bus system was taken and analysed, which are illustrated in the proceeding sub-sections.

\subsection{ATC enhancement performance on IEEE 24 bus system}

The performance of IEEE 24 test bus system for ATC enhancement by SAPSO is shown in table 1. The tabulation provides the information regarding the line number, the connection from the bus and to the bus and the

Table 1. Performance of ATC enhancement on IEEE 24 test bus system.

\begin{tabular}{|c|c|c|c|c|c|c|c|c|c|}
\hline \multirow[b]{2}{*}{ TCSC } & \multirow[b]{2}{*}{ Connections } & \multicolumn{2}{|c|}{2 TCSCs } & \multicolumn{2}{|c|}{3 TCSCs } & \multicolumn{2}{|c|}{4 TCSCs } & \multicolumn{2}{|c|}{5 TCSCs } \\
\hline & & PSO & SAPSO & $P S O$ & SAPSO & PSO & SAPSO & PSO & SAPSO \\
\hline \multirow[t]{3}{*}{1} & Line no & 28 & 22 & 22 & 7 & 37 & 36 & 21 & 15 \\
\hline & From bus/ to bus & $16 / 17$ & $13 / 23$ & $13 / 23$ & $3 / 24$ & $20 / 23$ & $20 / 23$ & $12 / 23$ & $9 / 12$ \\
\hline & Compensation (p.u) $\times 10^{-3}$ & 0.17924 & -0.2629 & 0.7 & 0.06034 & -0.39509 & 0.28118 & -0.7 & 0.23769 \\
\hline \multirow[t]{3}{*}{2} & Line no & 38 & 37 & 38 & 22 & 36 & 22 & 38 & 22 \\
\hline & (From bus/ to bus) & $21 / 22$ & $20 / 23$ & $21 / 22$ & $13 / 23$ & $20 / 23$ & $13 / 23$ & $21 / 22$ & $13 / 23$ \\
\hline & Compensation $(\mathrm{p} . \mathrm{u}) \times 10^{-3}$ & 0.16065 & -0.626 & 0.7 & 0.54866 & -0.11985 & -0.2365 & -0.32637 & -0.26575 \\
\hline \multirow[t]{3}{*}{3} & Line no & 0 & 0 & 38 & 10 & 14 & 1 & 38 & 26 \\
\hline & From bus/ to bus & $0 / 0$ & $0 / 0$ & $21 / 22$ & $6 / 10$ & 9/11 & $1 / 2$ & $21 / 22$ & $15 / 21$ \\
\hline & Compensation (p.u) $\times 10^{-3}$ & 0 & 0 & 0.7 & -0.3373 & 0.52401 & -0.3673 & 0.7 & 0.050421 \\
\hline \multirow[t]{3}{*}{4} & Line no & 0 & 0 & 0 & 0 & 5 & 1 & 38 & 38 \\
\hline & From bus/ to bus & $0 / 0$ & $0 / 0$ & $0 / 0$ & $0 / 0$ & $2 / 6$ & $1 / 2$ & $21 / 22$ & $21 / 22$ \\
\hline & Compensation (p.u) $\times 10^{-3}$ & 0 & 0 & 0 & 0 & 0.0026373 & -0.22587 & -0.7 & -0.22355 \\
\hline \multirow[t]{3}{*}{5} & Line no & 0 & 0 & 0 & 0 & 0 & 0 & 38 & 38 \\
\hline & From bus/ to bus & $0 / 0$ & $0 / 0$ & $0 / 0$ & $0 / 0$ & $0 / 0$ & $0 / 0$ & $21 / 22$ & $21 / 22$ \\
\hline & Compensation (p.u) $\times 10^{-3}$ & 0 & 0 & 0 & 0 & 0 & 0 & 0.7 & 0.17726 \\
\hline \multicolumn{2}{|c|}{ ATC (MW) without TCSC } & 16.6 & 16.6 & 16.6 & 16.6 & 16.6 & 16.6 & 16.6 & 16.6 \\
\hline \multicolumn{2}{|c|}{ ATC (MW) with TCSC } & 184.6 & 503.3 & 134.2 & 244.6 & 201.4 & 572.9 & 107.7 & 499 \\
\hline
\end{tabular}


Table 2. Performance of ATC enhancement on IEEE 30 test bus system.

\begin{tabular}{|c|c|c|c|c|c|c|c|c|c|}
\hline \multirow[b]{2}{*}{ TCSC } & \multirow[b]{2}{*}{ Connections } & \multicolumn{2}{|c|}{2 TCSCs } & \multicolumn{2}{|c|}{3 TCSCs } & \multicolumn{2}{|c|}{4 TCSCs } & \multicolumn{2}{|c|}{5 TCSCs } \\
\hline & & PSO & SAPSO & PSO & SAPSO & PSO & SAPSO & PSO & SAPSO \\
\hline \multirow[t]{3}{*}{1} & Line no & 10 & 27 & 14 & 27 & 9 & 41 & 27 & 41 \\
\hline & From bus/ to bus & $6 / 8$ & $10 / 21$ & $9 / 10$ & $10 / 21$ & $6 / 7$ & $6 / 28$ & $10 / 21$ & $6 / 28$ \\
\hline & Compensation (p.u) $\times 10^{-3}$ & -0.09536 & -0.17 & -0.14138 & -0.17 & 0.17 & 0.17 & -0.16575 & 0.17 \\
\hline \multirow[t]{3}{*}{2} & Line no & 38 & 41 & 10 & 31 & 41 & 9 & 1 & 27 \\
\hline & (From bus/ to bus) & $27 / 30$ & $6 / 28$ & $6 / 8$ & $22 / 24$ & $6 / 28$ & $6 / 7$ & $1 / 2$ & $10 / 21$ \\
\hline & Compensation (p.u) $\times 10^{-3}$ & -0.049768 & 0.17 & -0.16447 & -0.17 & 0.17 & 0.14154 & 0.17 & -0.17 \\
\hline \multirow[t]{3}{*}{3} & Line no & 38 & 38 & 6 & 41 & 41 & 1 & 13 & 41 \\
\hline & From bus/ to bus & $21 / 22$ & $21 / 22$ & $2 / 6$ & $6 / 28$ & $6 / 28$ & $1 / 2$ & $9 / 11$ & $6 / 28$ \\
\hline & Compensation (p.u) $\times 10^{-3}$ & 0.7 & 0.7 & -0.054805 & -0.17 & -0.17 & 0.17 & 0.17 & 0.17 \\
\hline \multirow[t]{3}{*}{4} & Line no & 38 & 38 & 38 & 38 & 41 & 41 & 24 & 41 \\
\hline & From bus/ to bus & $21 / 22$ & $21 / 22$ & $21 / 22$ & $21 / 22$ & $6 / 28$ & $6 / 28$ & $19 / 20$ & $6 / 28$ \\
\hline & Compensation (p.u) $\times 10^{-3}$ & -0.7 & -0.7 & -0.7 & -0.7 & 0.17 & 0.17 & 0.15097 & 0.17 \\
\hline \multirow[t]{3}{*}{5} & Line no & 38 & 38 & 38 & 38 & 38 & 38 & 1 & 1 \\
\hline & From bus/ to bus & $21 / 22$ & $21 / 22$ & $21 / 22$ & $21 / 22$ & $21 / 22$ & $21 / 22$ & $1 / 2$ & $1 / 2$ \\
\hline & Compensation (p.u) $\times 10^{-3}$ & 0.7 & 0.7 & 0.7 & 0.7 & 0.7 & 0.7 & 0.10555 & 0.17 \\
\hline \multicolumn{2}{|c|}{ ATC (MW) without TCSC } & 72.7 & 72.7 & 72.7 & 72.7 & 72.7 & 72.7 & 72.7 & 72.7 \\
\hline \multicolumn{2}{|c|}{ ATC (MW) with TCSC } & 129.6 & 143.5 & 140.5 & 147.9 & 78.7 & 78.7 & 141.7 & 143.5 \\
\hline
\end{tabular}

compensation. The experimentation is done for a power system with TCSC and without TCSC. Accordingly, the line number of the two TCSCs in the first connection recommends 28 for PSO and 22 for SAPSO, whereas the compensation level is 0.17924 for PSO and -0.26293 for SAPSO. Similarly, the line numbers of 3 TCSC, 4 TCSC, and 5 TCSC for the first connections are 22 for PSO and 7 for SAPSO, 37 for PSO and 36 for SAPSO and 21 for PSO and 15 for SAPSO. The compensation of 3 TCSC, 4 TCSC and 5 TCSC connections are estimated as 0.7 for PSO and 0.06034 for SAPSO, -0.39509 for PSO and 0.28118 for
SAPSO and -0.7 for PSO and 0.23769 for SAPSO. The same procedure is carried out for five connections, and a similar output is obtained. Thus, the ATC enhancement without TCSC connection provides the normal ATC of basic load system. Subsequently, the percentage of ATC measurement with TCSC using the proposed SAPSO method is $172 \%$ enhanced than using conventional PSO for two TCSC connections. To the same extent, the percentage of ATC measurement with TCSC for the proposed SAPSO method for 3TCSC, 4TCSC and 5TCSC is $82 \%, 184 \%$, and $363 \%$ better than the conventional PSO.

Table 3. Performance of ATC enhancement on IEEE 57 test bus system.

\begin{tabular}{|c|c|c|c|c|c|c|c|c|c|}
\hline \multirow[b]{2}{*}{ TCSC } & \multirow[b]{2}{*}{ Connections } & \multicolumn{2}{|c|}{2 TCSCs } & \multicolumn{2}{|c|}{3 TCSCs } & \multicolumn{2}{|c|}{4 TCSCs } & \multicolumn{2}{|c|}{5 TCSCs } \\
\hline & & $P S O$ & SAPSO & PSO & SAPSO & PSO & SAPSO & PSO & SAPSO \\
\hline \multirow[t]{3}{*}{1} & Line no & 80 & 49 & 80 & 80 & 80 & 49 & 80 & 42 \\
\hline & From bus/ to bus & $9 / 55$ & $36 / 37$ & $9 / 55$ & $9 / 55$ & $9 / 55$ & $36 / 37$ & $9 / 55$ & $25 / 30$ \\
\hline & Compensation (p.u) $\times 10^{-3}$ & 0.5 & -0.5 & 0.5 & 0.5 & 0.5 & -0.5 & 0.5 & -0.49925 \\
\hline \multirow[t]{3}{*}{2} & Line no & 80 & 80 & 80 & 49 & 80 & 1 & 80 & 80 \\
\hline & (From bus/ to bus) & $9 / 55$ & $9 / 55$ & $9 / 55$ & $36 / 37$ & $9 / 55$ & $1 / 2$ & $9 / 55$ & $9 / 55$ \\
\hline & Compensation (p.u) $\times 10^{-3}$ & 0.5 & 0.5 & 0.5 & -0.5 & 0.5 & 0.5 & 0.5 & 0.5 \\
\hline \multirow[t]{3}{*}{3} & Line no & 41 & 41 & 80 & 1 & 80 & 1 & 80 & 49 \\
\hline & From bus/ to bus & $6 / 28$ & $6 / 28$ & $9 / 55$ & $1 / 2$ & $9 / 55$ & $1 / 2$ & $9 / 55$ & $36 / 37$ \\
\hline & Compensation (p.u) $\times 10^{-3}$ & 0.17 & 0.17 & 0.5 & 0.5 & 0.5 & -0.5 & 0.5 & -0.5 \\
\hline \multirow[t]{3}{*}{4} & Line no & 41 & 41 & 41 & 41 & 80 & 1 & 80 & 1 \\
\hline & From bus/ to bus & $6 / 28$ & $6 / 28$ & $6 / 28$ & $6 / 28$ & $9 / 55$ & $1 / 2$ & $9 / 55$ & $1 / 2$ \\
\hline & Compensation (p.u) $\times 10^{-3}$ & 0.17 & 0.17 & 0.17 & 0.17 & 0.5 & 0.5 & 0.5 & -0.5 \\
\hline \multirow[t]{3}{*}{5} & Line no & 1 & 1 & 1 & 1 & 1 & 1 & 80 & 80 \\
\hline & From bus/ to bus & $1 / 2$ & $1 / 2$ & $1 / 2$ & $1 / 2$ & $1 / 2$ & $1 / 2$ & $9 / 55$ & $9 / 55$ \\
\hline & Compensation (p.u) $\times 10^{-3}$ & 0.17 & 0.17 & 0.17 & 0.17 & 0.17 & 0.17 & 0.5 & -0.5 \\
\hline \multicolumn{2}{|c|}{ ATC (MW) without TCSC } & 3 & 3 & 3 & 3 & 3 & 3 & 3 & 3 \\
\hline \multicolumn{2}{|c|}{ ATC (MW) with TCSC } & 3 & 42.9 & 3 & 42.9 & 3 & 42.9 & 3 & 77.1 \\
\hline
\end{tabular}


Table 4. Statistical analysis of IEEE 24, IEEE 30 and IEEE 57 test bus systems.

\begin{tabular}{|c|c|c|c|c|c|c|}
\hline \multirow{2}{*}{$\begin{array}{l}\text { Bus } \\
\text { Statistics }\end{array}$} & \multicolumn{2}{|c|}{ IEEE 24 bus system } & \multicolumn{2}{|c|}{ IEEE 30 bus system } & \multicolumn{2}{|c|}{ IEEE 57 bus system } \\
\hline & PSO & SAPSO & PSO & SAPSO & PSO & SAPSO \\
\hline Best & 330.5 & 335.8 & 78.7 & 143 & 3 & 42.9 \\
\hline Worst & 86.2 & 117.4 & 75.7 & 75.7 & 3 & 3 \\
\hline Mean & 201.2 & 247.4 & 78.1 & 101.24 & 3 & 24.66 \\
\hline Median & 134.2 & 292.4 & 78.7 & 75.7 & 3 & 31.5 \\
\hline Standard deviation & 115.38 & 89.512 & 1.3416 & 35.083 & 0 & 20.313 \\
\hline
\end{tabular}

\subsection{ATC enhancement performance on IEEE 30 bus system}

The performance regarding the enhancement of ATC of the proposed SAPSO is shown in table 2. The experiment is carried out for basic load system without TCSC and the connection of the power system with TCSC. Further, it is done for 2TCSC, 3TCSC, 4TCSC and 5TCSC under five connections. The line number of 2TCSC is 10 for PSO and 27 for SAPSO. Moreover, the line number of 3 TCSC is 14 for PSO and 27 for SAPSO, 4 TCSC is 9 for PSO and 41 for
SAPSO, and 5 TCSC connection is 27 for PSO and 41 for SAPSO. The same procedure is carried out for five connections. Similarly, the compensation of the ATC enhancement of 2 TCSC is -0.09536 for PSO and -0.17 for SAPSO, 3 TCSC is -0.14138 for PSO and -0.17 for SAPSO, 4 TCSC connections is 0.17 for PSO and 0.17 for SAPSO, and 5 TCSC connection is -0.16575 for PSO and 0.17 for SAPSO. Ultimately, according to the aforementioned performance analysis, the percentage of ATC measurement of the proposed SAPSO for 2TCSC connection is $107 \%$ better than PSO; 3 TCSC is $52 \%$ better than PSO, 4
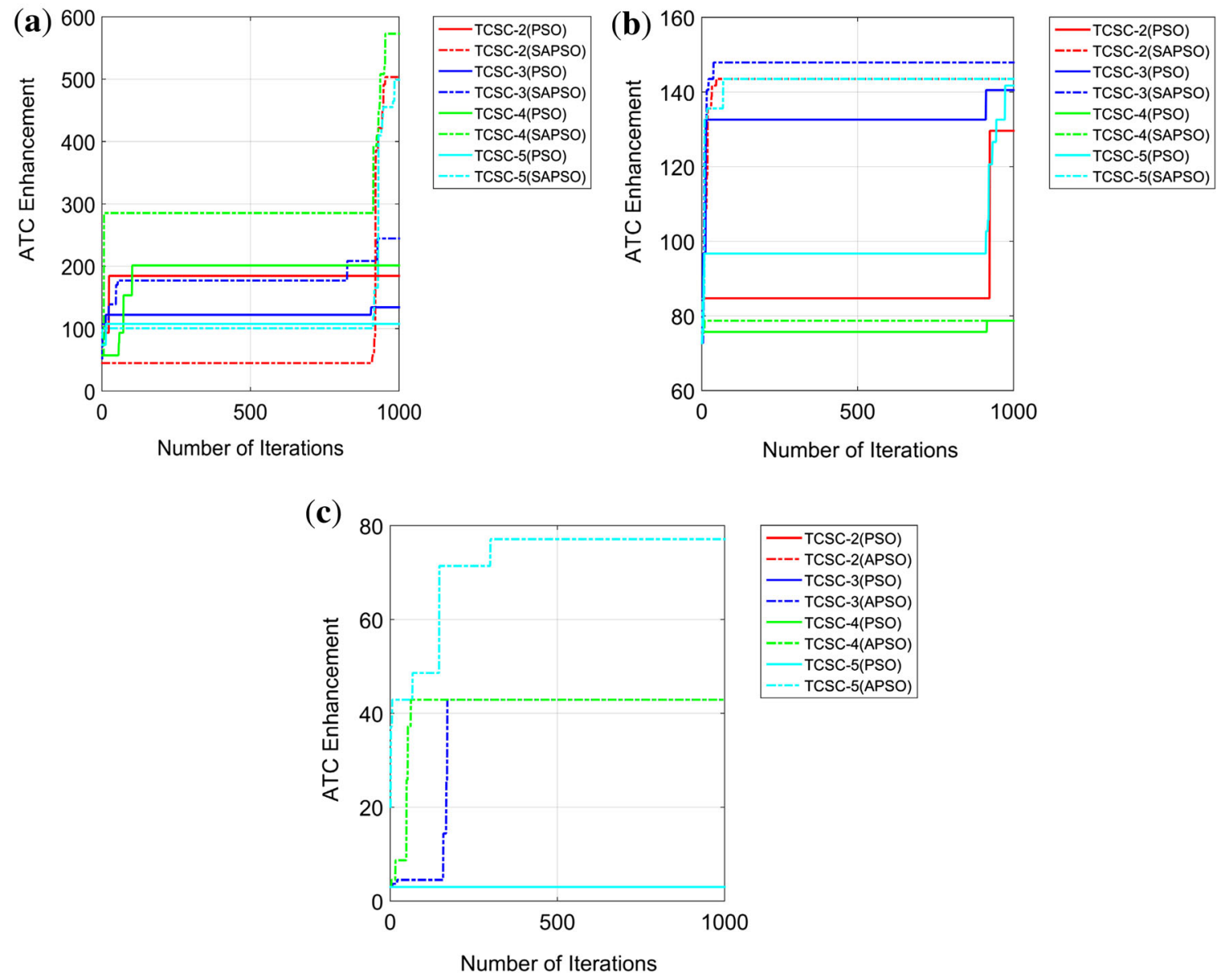

Figure 3. Demonstration of ATC enhancement for 1000 iterations on (a) IEEE 24 bus syestem, (b) IEEE 30 bus system and (c) IEEE 57 bus system. 
TCSC is same as PSO, and 5 TCSC connections are $12 \%$ better than PSO. Thus, from the overall analysis, the proposed SAPSO provides superior performance.

\subsection{ATC enhancement performance on IEEE 57 bus system}

The performance of ATC enhancement of IEEE 57 test bus system is shown in table 3. First, the line number of each TCSC connection for conventional and proposed PSO is determined. The line number for 2TCSC, 3TCSC, 4TCSC and 5TCSC connections are 80 for PSO and 49 for SAPSO, 80 for PSO and 80 for SAPSO, 80 for PSO and 49 for SAPSO and 80 for PSO and 42 for SAPSO. Similarly, the first set of compensation for 2TCSC, 3TCSC, 4TCSC and 5TCSC connections is 0.5 for PSO and -0.5 for SAPSO, 0.5 for PSO and 0.5 for SAPSO, 0.5 for PSO and -0.5 for SAPSO and 0.5 for PSO and 0.5 for PSO and -0.49925 for SAPSO. Accordingly, the ATC enhancement of the system with and without TCSC is performed individually. The ATC enhancement without TCSC connection is same for both PSO and SAPSO, whereas the enhancement of ATC with TCSC connection for 2TCSC, 3TCSC, 4TCSC and 5TCSC connections for proposed SAPSO are far higher than the conventional PSO algorithm.

\subsection{Statistical analysis}

The statistical analysis of IEEE 24, IEEE 30 and IEEE 57 bus system is shown in table 4 . The best performance of SAPSO on IEEE 24 test bus system is $1.6 \%$ better than PSO, and IEEE 30 test bus system is $81 \%$ better than PSO, whereas the SAPSO of IEEE 57 test bus system is far better than conventional PSO. Similarly, the worst, mean, median and standard deviation performance of ATC enhancement on IEEE 24, IEEE 30 and IEEE 57 test bus system of proposed SAPSO is higher than conventional PSO algorithm.

The graphical representation of the appearance of ATC enhancement for 1000 iterations on IEEE 24, IEEE 30 and IEEE 57 test bus system is shown in figure 3. In IEEE 24 bus system, ATC measurement of 2TCSC, 3 TCSC, 4 TCSC and 5 TCSC connection of SAPSO is higher than the conventional PSO. On the other hand, the ATC measurement of entire TCSC connection of IEEE 30 test bus system by SAPSO is higher than the conventional PSO. Finally, the IEEE 57 test bus system also provides higher ATC. Thus, it proves that the proposed SAPSO is better than conventional PSO for enhancing ATC in power system.

\section{Conclusion}

Generally, ATC should be enhanced to improve the reliability of the power system. However, ATC is reduced in some cases when the power system is affected by usage of overloaded circuits and buses with limited voltage. In this study, SAPSO algorithm was proposed in order to optimize the maximum ATC in power transmission system. Subsequently, this study has used TCSC devices instead of FACTS devices. The simulation setup was carried out on IEEE 24, IEEE 30 and IEEE 57 test bus systems. Further, the statistical analysis of the proposed SAPSO method conducted on the three test bus system was compared with the conventional PSO algorithm. The result obtained from the comparison has proved that the SAPSO enhances ATC measurement with maximum improvement than conventional PSO algorithm.

\section{References}

[1] Gyugyi L 1992 A unified power flow control concept for flexible AC transmission systems. IEEE Proceedings of Generation, Transmission and Distribution, Pittsburgh, USA, 139(4): 323-331

[2] Swamy S M, Rajakumar B R and Valarmathi I R 2013 Design of hybrid wind and photovoltaic power system using opposition-based genetic algorithm with Cauchy mutation. In: Proceedings of IET Chennai 4th International Conference on Sustainable Energy and Intelligent Systems (SEISCON 2013), Chennai, pp. 504-510

[3] Ejebe G C, Tong J, Waight J G, Frame J G, Wang X and Tinney W F 1998 Available transfer capability calculations. IEEE Trans. Power Syst. 13(4): 1521-1527

[4] Padmavathi S V, Sahu S and Jayalakshmi A 2012 Available transfer capability enhancement by using Particle Swarm Optimization algorithm based FACTS allocation. In: 2012 Asia Pacific Conference on Postgraduate Research in Microelectronics and Electronics, Hyderabad, pp. 184-187

[5] Yadav N 2015 Genetic algorithm with dual mutation probabilities for TCSC based ATC enhancement. In: Proceedings of Sixth International Conference on Advances in Computing, Control, and Telecommunication Technologies - ACT

[6] Ali E S and Abd-Elazim S M 2012 Coordinated design of PSSs and TCSC via bacterial swarm optimization algorithm in a multimachine power system. Int. J. Elec. Power. 36(1): 84-92

[7] Farahmand H, Rashidi-Nejad M and Fotuhi-Firoozabad M 2004 Implementation of FACTS devices for ATC enhancement using RPF technique. In: Power Engineering, 2004 LESCOPE-04. 2004 Large Engineering systems Conference, pp. $30-35$

[8] Transmission Transfer Capability Task Force 1996 Available transfer capability Definitions and determination, North American Electric Reliability Council, New Jersey

[9] Galiana F D, Almeida K, Toussaint M, Griffin J, Atanackovic D, Ooi B T and McGillis D T 1996 Assessment and control of the impact of FACTS devices on power system performance. IEEE Trans. Power Syst. 11(4): 1931-1936

[10] Nireekshana T, Kesava Rao G and Siva Naga Raju S 2012 Enhancement of ATC with FACTS devices using Real-code Genetic Algorithm. Int. J. Elec. Power 43(1): 1276-1284 
[11] Acharya N and Mithulananthan N 2007 Locating series FACTS devices for congestion management in deregulated electricity markets. Electr. Pow. Syst. Res. 77(3-4): 352-360

[12] Canizares C A and Z T Faur 1999 Analysis of SVC and TCSC controllers in voltage collapse. IEEE Trans. Power Syst. 14(1): 158-165

[13] Ibraheem N and Yadav N K 2011 Implementation of FACTS Device for Enhancement of ATC Using PTDF. Int. J. Comput. Electr. Eng. 3(3): 343-348

[14] Mahmoudian M and Yousefi G R ATC improvement and losses estimation considering dynamic transmission line ratings. In: 20th Iranian Conference on Electrical Engineering (ICEE2012), pp. 404-409

[15] Hingorani N G and Gyugyi L 2000 Understanding facts, concepts and technology of flexible AC transmission systems. New York: IEEE

[16] Bavithra K, Charles Raja S and Venkatesh P 2016 Optimal setting of FACTS devices using particle swarm optimization for ATC enhancement in deregulated power system. In: 4th IFAC Conference on Advances in Control and Optimization of Dynamical Systems ACODS 2016, 49(1): 450-455

[17] Bavithra K, Charles Raja S and Venkatesh P 2016 Optimal setting of FACTS devices using particle swarm optimization for ATC enhancement in deregulated power system. IFACPapersOnLine 49(1): 450-455

[18] Nireekshana T, Kesava Rao G and Siva Naga Raju S 2012 Enhancement of ATC with FACTS devices using Real-code Genetic Algorithm. Int. J. Electr. Power 43(1): 1276-1284

[19] Gupta A and Kumar A 2016 Impact of TCSC installation on ATC in a system incorporating wind and hydro generations. Proced. Technol. 25: 743-750

[20] Farahmand H, Rashidinejad M, Mousavi A, Gharaveisi A A, Irving M R and Taylor G A 2012 Hybrid mutation particle swarm optimisation method for available transfer capability enhancement. Electr. Power Energy Syst. 42(1): 240-249

[21] Karimi-Nasab M, Modarres M and Seyedhoseinic S M 2015 A self-adaptive PSO for joint lot sizing and job shop scheduling with compressible process times. Appl. Soft Comput. 27: 137-147

[22] Zuo X, Zhang G and Tan W 2014 Self-adaptive learning PSO-based deadline constrained task scheduling for hybrid IaaS cloud. IEEE Trans. Autom. Sci. Eng. 11(2): 564-573

[23] Rashidinejad M, Farahmand H, Fotuhi-Firuzabad M and Gharaveisi A A 2008 ATC enhancement using TCSC via artificial intelligent techniques. Electr. Power Syst. Res. 78(1): 11-20

[24] Khaburi M A and Haghifam M R 2010 A probabilistic modeling based approach for total transfer capability enhancement using FACTS devices. Int. J. Electr. Power. 32(1): 12-16

[25] Hiraki Y, Hiraiwa T and Iwamoto S 2012 NAS battery system design and allocation for improvement of transient stability ATC. In: 2012 10th International Power \& Energy Conference (IPEC), Chicago, pp. 190-195

[26] Satoh T, Tanaka H and Iwamoto S 2007 ATC Improvement by phase shifter application considering dynamic rating, In: Power Symposium, 2007 NAPS '07 39th North American, Las Cruces, pp. 528-533

[27] Jain T, Singh S N and Srivastava S C Dynamic ATC enhancement through optimal placement of FACTS controllers. Electr. Power Syst. Res. 79(11): 1473-1482

[28] De A, Mamanduru V K R, Gunasekaran A, Subramanian N and Tiwari M K 2016 Composite particle algorithm for sustainable integrated dynamic ship routing and scheduling optimization. Comput. Ind. Eng. 96: 201-215

[29] Alexandridis A, Chondrodima E and Sarimveis H 2016 Cooperative learning for radial basis function networks using particle swarm optimization. Appl. Soft Comput. 49: 485-497

[30] De A, Kumar S, Gunasekaran A and Tiwari M K 2017 Sustainable maritime inventory routing problem with time window constraints. Eng. Appl. Artif. Intell. 61: 77-95

[31] Soleimani H and Kannan G 2015 A hybrid particle swarm optimization and genetic algorithm for closed-loop supply chain network design in large-scale networks. Appl. Math. Model. 39(14): 3990-4012

[32] De A, Awasthi A and Tiwari M K 2015 Robust formulation for optimizing sustainable ship routing and scheduling problem. IFAC-PapersOnLine. 48(3): 368-373

[33] Li C-Y and Liu C-W 2002 A new algorithm for available transfer capability computation. Int J. Elec. Power. 24(2): 159-166

[34] Vaithilingam C and Kumudini Devi R P 2013 Available transfer capability estimation using Support Vector Machine. Int. J. Elec. Power. 47: (May): 387-393

[35] Sheng $\mathrm{H}$ and Chiang H-D 2014 CDFLOW: A practical tool for tracing stationary behaviors of general distribution networks. IEEE Trans. Power Syst. 29(3): 1365-1371 